\title{
A simple proof of magnetic and electric Aharonov-Bohm effects
}

\author{
G.Eskin, Department of Mathematics, UCLA, \\ Los Angeles, CA 90095-1555, USA. E-mail: eskin@math.ucla.edu
}

August 31, 2018

\begin{abstract}
Magnetic Aharonov-Bohm effect (AB effect) was studied in hundreds of papers starting with the seminal paper of Aharonov and Bohm $[\mathrm{AB}]$ published in 1959. We give a new proof of the magnetic Aharonov-Bohm effect without using the scattering theory and the theory of inverse boundary value problems. We consider separately the cases of one and several obstacles. The electric AB effect was studied much less. We give the first proof of the electric $A B$ effect in domains with moving boundaries. When the boundary does not move with the time the electric $\mathrm{AB}$ effect is absent.
\end{abstract}

\section{Introduction.}

Let $\Omega_{1}$ be a bounded domain in $\mathbf{R}^{2}$, called the obstacle. Consider the timedependent Schrödinger equation in $\left(\mathbf{R}^{2} \backslash \bar{\Omega}_{1}\right) \times(0, T)$ :

$$
-i h \frac{\partial u}{\partial t}+\frac{1}{2 m} \sum_{j=1}^{n}\left(-i h \frac{\partial}{\partial x_{j}}-\frac{e}{c} A_{j}(x)\right)^{2} u+e V(x) u=0,
$$

where $n=2$,

$$
\begin{gathered}
\left.u\right|_{\partial \Omega_{1} \times(0, T)}=0, \\
u(x, 0)=u_{0}(x), \quad x \in \mathbf{R}^{2} \backslash \Omega_{1},
\end{gathered}
$$


and the electromagnetic potentials $A(x), V(x)$ are independent of $t$. The equation (1.1) describes an electron in $\left(\mathbf{R}^{2} \backslash \bar{\Omega}\right) \times(0, T)$, more precisely, $|u(x, t)|^{2}$ is a probability density of finding the electron in an infinitesimal neighborhood of $x$. Let

$$
\alpha=\frac{e}{h c} \int_{\gamma} A(x) \cdot d x
$$

be the magnetic flux, where $\gamma$ is a simple closed contour containing $\Omega_{1}$. In the seminal paper $[\mathrm{AB}]$ Aharonov and Bohm discovered that even when the magnetic field $B(x)=\operatorname{curl} A=0$ in $\mathbf{R}^{2} \backslash \bar{\Omega}_{1}$, the magnetic potential $A$ affects the electron in $\mathbf{R}^{2} \backslash \bar{\Omega}_{1}$ if $\alpha \neq 2 \pi n, \forall n \in Z$. This phenomenon is called the Aharonov-Bohm effect, where we say that the Aharonov-Bohm effect takes place if there are solutions $u(x, t)$ of (1.1) for which a physical quantity such as the probability density $|u(x, t)|^{2}$ or probability current $\operatorname{Re}\left(\overline{u(x, t)}\left(-i \frac{\partial}{\partial x}\right) u(x, t)\right)$ depends on the gauge equivalence class of the magnetic potential.

The purpose of the present paper is to give a simple proof of the AB effect without using the scattering theory or the theory of inverse boundary value problems.

Aharonov and Bohm proposed a physical experiment to test the $\mathrm{AB}$ effect. The experimental proof of $\mathrm{AB}$ effect was not easy to achieve in the way that is free of any controversy. This was accomplished by Tonomura et al [ $\mathrm{T}$ et al]. In the same paper [AB] Aharonov and Bohm gave a mathematical proof of $\mathrm{AB}$ effect in the case when the obstacle is reduced to a point: $\Omega_{1}=\{0\}$. They explicitly constructed the scattering amplitude and showed that the scattering cross-section is influenced by the magnetic flux modulo $2 \pi n, n \in$ Z. Later Ruijsenaars $[R]$ proved the same result in the case when the obstacle is a circle. The next important step was done by Nicoleau $[\mathrm{N}]$ who found a way to compute the integrals

$$
\exp \left(\frac{i e}{h c} \int_{-\infty}^{\infty} A\left(x_{0}+t \omega\right) \cdot \omega d t\right)
$$

and

$$
\int_{-\infty}^{\infty} V\left(x_{0}+t \omega\right) d t
$$

knowing the scattering operator. In (1.5), (1.6) $n=2$ or $n=3$ and $x=x_{0}+t \omega, t \in \mathbf{R}$, is any straight line that does not intersect the obstacles. When the obstacle $\Omega$ is convex, Nicoleau [N] used (1.5), (1.6) and 
the $X$-ray transform to determine $B=\operatorname{curl} A, V(x)$ and the magnetic flux $\alpha(\bmod 2 \pi m)$ for $n=2$, i.e. he solved the inverse scattering problem.

When considering the Aharonov-Bohm effect, the magnetic field $B(x)=0$, and therefore one needs only a finite number of integrals of the form (1.5) to determine the magnetic fluxes modulo $2 \pi p, p \in \mathbf{Z}$. In this case the $X$-ray transform is not needed. Still the recovery of integrals (1.5) from the scattering operator is quite complicated. The motivation of the present work was to find an easier proof of the AB effect that does not involve the scattering theory.

In [W1] Weder considered the case when $n=2$ and $V(x)=0$ and he recovered integrals (1.5) from the scattering operator in a larger class of potentials then in $[\mathrm{N}]$. He also considered the case when the obstacle is reduced to a point as in the original Aharonov-Bohm paper $[\mathrm{AB}]$.

In a series of papers [E3], [E4], [E5], [E6] the inverse boundary value problems for the Schrödinger equations with time-independent electromagnetic potentials were studied. The most complete result was obtained in [E6] where an arbitrary number of obstacles (not necessary convex) was considered, and it was proven that the boundary data determine the gauge equivalent class of electromagnetic potentials. In particular, the magnetic AB effect was proven. In [E6] and [E3] the case of Yang-Mills potentials generalizing electromagnetic potentials was also considered.

It is well known that the inverse boundary value problem can be reduced to the inverse scattering problem, and vice versa, in the case when the magnetic and electric potentials have a compact support.

In the case of the magnetic $A B$ effect it is natural to assume that the magnetic field $B(x)$ has a compact support. The electric potential does not affect the magnetic $\mathrm{AB}$ effect and, for the simplicity, we can assume that $V(x)$ has a compact support too, or even $V(x)$ equal to zero. If $B(x)$ has a compact support and if $n \geq 3$, or $n=2$ and the total magnetic flux is zero, then one can find a magnetic potential $A(x)$ with compact support such that $B(x)=\operatorname{curl} A(x)$ (cf. [E4], $\S 1$ ). In this case the results of [E6] lead to the solution of the inverse scattering problem and, in particular, to the proof of the magnetic AB effect.

In [BW1] Ballesteros and Weder recovered integrals (1.5), (1.6) from the scattering operator for $n=3$ in a class of potentials larger than in $[\mathrm{N}]$ with less requirements on the smoothness. They also proved in [BW1] the AB effect for some quite restrictive class of obstacles in $\mathbf{R}^{3}$. When the electric potential has a compact support their results were preceded by [E6] where a 
general class of obstacles was considered.

When $n=2$ and the total flux is not zero the magnetic potential is $O\left(\frac{1}{|x|}\right)$ even when $B(x)$ has a compact support. In this case the inverse scattering problems become more difficult. This case was treated in [EIO] (see also $[\mathrm{EI}]$ ). The proofs in [EIO], [EI] use results of $[\mathrm{N}]$ (or [W1]) and also the results of Yafaev and Roux-Yafaev ([RY1], [RY2], [Y]) on the singularities of the scattering matrix in the case of long range magnetic potentials.

All previous papers considered the case of electromagnetic potentials independent of the time. There are only two papers [E1] and [W3] that consider inverse problems for the Schrödinger equations with time-dependent potentials but [W3] has no relation to the $\mathrm{AB}$ effect and [E1] is the only paper studying the inverse boundary value problem for the time-dependent electromagnetic potentials. In particular, [E1] gives the proof of the combined electric and magnetic AB effect.

Now we shall describe the content of the present paper.

We start in $\S 3$ (subsection 3.1) with the proof of the magnetic AB effect in the case of one obstacle in $\mathbf{R}^{2}$ by constructing solutions of nonstationary Schrödinger equations depending on a large parameter. We construct such solutions in two steps: first we construct a geometric optics solutions of the wave equation concentrated in a small neighborhood of a ray and then we use the Kannai's formula (cf. [K]) that transform the solution of the wave equation into the solution of the Schrödinger equation. We use these solutions to prove the following theorem

Theorem 1.1. There exists a solution $u(x, t)$ of the Schrödinger equation (1.1) in $\left(\mathbf{R}^{2} \backslash \bar{\Omega}_{1}\right) \times(0, T)$ with the boundary condition (1.2) and some highly oscillating initial data (1.3) such that

$$
|u(x, t)|^{2}=2 \sin ^{2} \frac{\alpha}{2}+O(\varepsilon)
$$

in an $\varepsilon$-neighborhood of some point, where $\varepsilon$ is small and $\alpha$ is the magnetic flux (1.4), $\alpha \neq 2 \pi n, \forall n \in \mathbf{Z}$. Therefore, the probability density $|u(x, t)|^{2}$ depends on the magnetic flux $\alpha$, i.e. the AB effect holds. Moreover $|u(x, t)|^{2}$ determines $\alpha(\bmod 2 \pi n, n \in \mathbf{Z})$ up to a sign.

In a short subsection 3.2 we extend Theorem 1.1 to the three-dimensional case.

In subsection 3.3 we prove a theorem similar to Theorem 1.1 in the case of several obstacles in $\mathbf{R}^{2}$ (see Theorem 3.1 below). The case of several 
obstacles requires the construction of the geometric optics solutions for the wave equation concentrated in the neighborhood of broken rays, i.e. rays reflected at the obstacles.

The use of broken rays allows to treat the case when obstacles are close to each other and the treatment of the cluster of obstacles as one obstacle may miss the $\mathrm{AB}$ effect. It is mentioned in the Remark 3.1 that the broken rays are used sometimes in the case of one obstacle too.

In $\S 4$ the electric $\mathrm{AB}$ effect is considered, assuming that the magnetic potential $A=0$ and the electric field $E=\nabla V(x, t)=0$ in the domain $D \subset \mathbf{R}^{n} \times[0, T]$. The electric AB effect is studied much less than the magnetic AB effect. When the domain $D$ has the form $\Omega \times(0, T)$, where $\Omega \subset \mathbf{R}^{2}$ is connected, then $E=\nabla V(x, t)=0$ in $D$ implies that $V(x, t)=V(t)$. Such electric potentials are gauge equivalent to zero electric potential, i.e. there is no electric AB effect. It is not surprising that there was neither mathematical nor experimental proof of electric $\mathrm{AB}$ effect in such domains. To get an electric $\mathrm{AB}$ effect one needs to consider domains $D$ such that the topology of the intersection $D \cap\left\{t=t_{0}\right\}$ changes with $t_{0}$, i.e. the boundary of $D$ moves with the time. Such domains are considered in $\S 4$ and the following theorem is proven

Theorem 1.2. Let $u(x, t)$ be the solution of the Schrödinger equation

$$
i h \frac{\partial u}{\partial t}+\frac{h^{2}}{2 m} \Delta u-e V(x, t) u=0, \quad(x, t) \in D
$$

with zero boundary conditions and nonzero initial condition. Let $v(x, t)$ be the solution of (1.8) with $V(x, t)=0$ and the same initial and boundary conditions as $u(x, t)$. Suppose that $E=\frac{\partial V(x, t)}{\partial x}=0$ in $D$ and that $V(x, t)$ is not gauge equivalent to zero potential. Then there exists a domain $D$ such that the probability densities $|u(x, t)|^{2}$ and $|v(x, t)|^{2}$ differ in $D$, i.e. the impact of the electric potential $V(x, t)$ is different from the impact of the zero potential.

This proves the electric AB effect.

\section{The magnetic AB effect}

Let $\Omega_{1}, \ldots, \Omega_{m}$ be smooth obstacles in $\mathbf{R}^{n}$. Assume that $\bar{\Omega}_{j} \cap \bar{\Omega}_{k} \neq 0$ if $j \neq k$. Consider the Schrödinger equation (1.1) in $\left(\mathbf{R}^{n} \backslash \bar{\Omega}\right) \times(0, T), n \geq 2$, where 
$\Omega=\cup_{j=1}^{m} \Omega_{j}$,

$$
\left.u\right|_{\partial \Omega \times(0, T)}=0
$$

and (1.3) holds in $\mathbf{R}^{n} \backslash \Omega$.

Let $B(x)=\operatorname{curl} A(x)$ be the magnetic field, $n=2$ or 3 . In the case $n>3$ we consider $B(x)$ as the differential of the form $\sum_{j=1}^{n} A_{j}(x) d x_{j}$.

In this paper we assume that $B(x)=0$ in $\mathbf{R}^{n} \backslash \bar{\Omega}$, i.e. the magnetic field $B(x)$ is shielded inside $\Omega$. For the simplicity, we assume that $V(x)$ has a compact support.

Denote by $G\left(\mathbf{R}^{n} \backslash \Omega\right)$ the group of $C^{\infty}$ complex-valued functions $g(x)$ such that $|g(x)|=1$ in $\mathbf{R}^{n} \backslash \Omega$ and

$$
\begin{array}{r}
g(x)=1+O\left(\frac{1}{|x|}\right) \text { for }|x|>R \text { if } n \geq 3 \\
g(x)=e^{i p \theta(x)}\left(1+O\left(\frac{1}{|x|}\right)\right) \text { for }|x|>R \text { if } n=2 .
\end{array}
$$

Here $p$ is an arbitrary integer, $0 \in \Omega$ and $\theta(x)$ is the polar angle of $x$. The difference between the cases $n=2$ and $n \geq 3$ is the consequence of the fact that the set $\{x:|x|>R\}$ is simply connected when $n \geq 3$ and it is not simply-connected when $n=2$. We call $G\left(\mathbf{R}^{n} \backslash \Omega\right)$ the gauge group. If $u^{\prime}(x, t)=g^{-1}(x) u(x, t)$ then $u^{\prime}(x, t)$ satisfies the Schrödinger equation (1.1) with electromagnetic potentials $\left(A^{\prime}(x), V^{\prime}(x)\right)$, where $V^{\prime}(x)=V(x)$ and

$$
\frac{e}{c} A^{\prime}(x)=\frac{e}{c} A(x)+i h g^{-1}(x) \frac{\partial g(x)}{\partial x} .
$$

We shall call magnetic potentials $A^{\prime}(x)$ and $A(x)$ gauge equivalent if there exists $g(x) \in G\left(\mathbf{R}^{n} \backslash \Omega\right)$ such that (2.2) holds.

We shall describe all gauge equivalence classes of magnetic potentials when $B=\operatorname{curl} A=0$ in $\mathbf{R}^{n} \backslash \Omega$.

It is easy to show (see, for example, $\S 4$ in [E1]) that $A(x)$ and $A^{\prime}(x)$ are gauge equivalent iff for any closed contour $\gamma$ in $\mathbf{R}^{n} \backslash \bar{\Omega}$ we have

$$
\frac{e}{h c} \int_{\gamma} A(x) \cdot d x-\frac{e}{h c} \int_{\gamma} A^{\prime}(x) \cdot d x=2 \pi p,
$$

where $p \in \mathbf{Z}$. 
Since curl $A=0$ in $\mathbf{R}^{n} \backslash \bar{\Omega}$ the integral $\int_{\gamma} A(x) \cdot d x$ depends only on homotopy class of $\gamma$ in $\mathbf{R}^{n} \backslash \Omega$.

Consider the case of one obstacle $\Omega_{1}$ in $\mathbf{R}^{2}$. Let $\gamma_{1}$ be a simple closed contour in $\mathbf{R}^{2} \backslash \bar{\Omega}_{1}$ containing $\Omega_{1}$. Any closed contour $\gamma$ in $\mathbf{R}^{2} \backslash \bar{\Omega}_{1}$ is homotopic to $m \gamma_{1}$, where $m \in \mathbf{Z}$. Therefore the gauge equivalent class in $G\left(\mathbf{R}^{2} \backslash \Omega_{1}\right)$ is determined by the magnetic flux $\frac{e}{h c} \int_{\gamma_{1}} A(x) \cdot d x$ modulo $2 \pi p, p \in \mathbf{Z}$.

In the case of several obstacles $\Omega_{1}, \ldots, \Omega_{m}$ in $\mathbf{R}^{2}$ denote by $\gamma_{j}, 1 \leq j \leq m$, a simple closed curve encircling $\Omega_{j}$ only. Let

$$
\alpha_{j}=\frac{e}{h c} \int_{\gamma_{j}} A \cdot d x
$$

be the corresponding magnetic flux.

Any closed contour $\gamma$ in $\mathbf{R}^{2} \backslash \bigcup_{j=1}^{m} \bar{\Omega}_{j}$ is homotopic to $\sum_{j=1}^{m} n_{j} \gamma_{j}$, $n_{j} \in \mathbf{Z}$. Therefore the numbers $\alpha_{j}\left(\bmod 2 \pi p_{j}\right), p_{j} \in Z, j=1, \ldots, m$, determine the gauge equivalence class of $A(x)$. Analogously, in the case $m \geq 1$ obstacles in $\mathbf{R}^{n}, n \geq 3$, any closed contour in $\mathbf{R}^{n} \backslash \bar{\Omega}$ is homotopic to $\sum_{j=1}^{r} n_{j} \beta_{j}$, where $\left\{\beta_{1}, \ldots, \beta_{r}\right\}$ is the basis of the homology group of $\mathbf{R}^{n} \backslash \bar{\Omega}, n_{j} \in \mathbf{Z}$. Therefore $A(x)$ and $A^{\prime}(x)$ are gauge equivalent iff

$$
\frac{e}{h c} \int_{\beta_{j}} A(x) \cdot d x-\frac{e}{h c} \int_{\beta_{j}} A(x) \cdot d x=2 \pi p_{j}, p_{j} \in \mathbf{Z},
$$

for all $1 \leq j \leq r$.

Any two magnetic potentials belonging to the same gauge equivalence class represent the same physical reality and can not be distinguished in any physical experiment.

Consider the probability density $|u(x, t)|^{2}$. It has the same value for any representative of the same gauge equivalence class since $\left|g^{-1}(x) u(x, t)\right|^{2}=$ $|u(x, t)|^{2}$.

To prove the $\mathrm{AB}$ effect it is enough to show that for some $u(x, t)$ the probability density $|u(x, t)|^{2}$ changes when we change the gauge equivalence class.

\section{The proof of the magnetic AB effect}

\subsection{The case of one obstacle in $\mathbf{R}^{2}$}

Consider the Schrödinger equation (1.1) in $\left(\mathbf{R}^{2} \backslash \Omega_{1}\right) \times(0, T)$ with the boundary condition (1.2) and the initial condition (1.3). 
Let $w(x, t)$ be the solution of the wave equation

$$
\frac{h^{2}}{2 m} \frac{\partial^{2} w}{\partial t^{2}}+H w=0 \text { in }\left(\mathbf{R}^{2} \backslash \Omega_{1}\right) \times(0,+\infty)
$$

with the boundary condition

$$
\left.w\right|_{\partial \Omega_{1} \times(0,+\infty)}=0
$$

and the initial conditions

$$
w(x, 0)=u_{0}(x), \quad \frac{\partial w(x, 0)}{\partial t}=0, \quad x \in \mathbf{R}^{2} \backslash \Omega_{1},
$$

i.e. $w(x, t)$ is even in $t$. Here

$$
H=\frac{1}{2 m}\left(-i h \frac{\partial}{\partial x}-\frac{e}{c} A\right)^{2}+e V(x) .
$$

There is a formula relating $u(x, t)$ and $w(x, t)$ (cf. Kannai $[\mathrm{K}])$ :

$$
u(x, t)=\frac{e^{-i \frac{\pi}{4}} \sqrt{m}}{\sqrt{2 \pi h t}} \int_{-\infty}^{\infty} e^{\frac{i m x_{0}^{2}}{2 h t}} w\left(x, x_{0}\right) d x_{0} .
$$

We shall consider solutions of (3.1) such that

$$
|w(x, t)| \leq C(1+|t|)^{m}, \quad\left|\frac{\partial^{r} w(x, t)}{\partial t^{r}}\right| \leq C_{r}(1+|t|)^{m}, \quad \forall r \geq 1 .
$$

Let $\chi_{0}(t) \in C_{0}^{\infty}\left(\mathbf{R}^{1}\right), \chi_{0}(-t)=\chi_{0}(t), \chi_{0}(t)=1$ for $|t|<\frac{1}{2}, \chi_{0}(t)=0$ for $|t|>1$. We define the integral (3.4) as the limit of

$$
\frac{e^{-i \frac{\pi}{4}} \sqrt{m}}{\sqrt{2 \pi h t}} \int_{-\infty}^{\infty} \chi_{0}\left(\varepsilon x_{0}\right) e^{\frac{i m x_{0}^{2}}{2 h t}} w\left(x, x_{0}\right) d x_{0}
$$

as $\varepsilon \rightarrow 0$, and we shall show that this limit exists for any $w\left(x, x_{0}\right)$ satisfying (3.5). Substitute the identity

$$
\left(\frac{h t}{i m x_{0}} \frac{\partial}{\partial x_{0}}\right)^{M} e^{\frac{i m x_{0}^{2}}{2 h t}}=e^{\frac{i m x_{0}^{2}}{2 h t}}, \quad \forall M
$$

in (3.6) and integrate by parts in (3.6) for $\left|x_{0}\right|>1$. If $M \geq m+2$ we get an absolutely integrable function of $x_{0}$ and therefore we can pass to the limit when $\varepsilon \rightarrow 0$. 
Note that

$$
\left(-i h \frac{\partial}{\partial t}+H\right) u(x, t)=\frac{e^{-i \frac{\pi}{4}} \sqrt{m}}{\sqrt{2 \pi h t}} \int_{-\infty}^{\infty} e^{\frac{i m x_{0}^{2}}{2 h t}}\left(\frac{h^{2}}{2 m} \frac{\partial^{2}}{\partial x_{0}^{2}}+H\right) w\left(x, x_{0}\right) d x_{0},
$$

Note also that

$$
u(x, 0)=\lim _{t \rightarrow 0} \frac{e^{-i \frac{\pi}{4}} \sqrt{m}}{\sqrt{2 \pi h t}} \int_{-\infty}^{\infty} e^{\frac{i m x_{0}^{2}}{2 h t}} w\left(x, x_{0}\right) d x_{0}=w(x, 0) .
$$

Therefore $u(x, t)$ satisfies (1.1), (1.2), (1.3) if $w(x, t)$ satisfies (3.1), (3.2), (3.3).

We shall construct geometric optics type solutions of (3.1), (3.2), (3.3) and then use the formula (3.4) to obtain solutions of the Schrödinger equation.

We shall look for $w(x, t)$ in the form

$$
w_{N}(x, t)=e^{i \frac{m k}{h}(x \cdot \omega-t)} \sum_{p=0}^{N} \frac{a_{p}(x, t)}{(i k)^{p}}+e^{i \frac{m k}{h}(x \cdot \omega+t)} \sum_{p=0}^{N} \frac{b_{p}(x, t)}{(i k)^{p}}
$$

where $k$ is a large parameter and $\omega$ is a unit vector, i.e. $|\omega|=1$.

Substituting (3.7) into (3.1) and equating equal powers of $k$ we get

$$
\begin{array}{r}
h a_{0 t}(x, t)+h \omega \cdot a_{0 x}(x, t)-i \omega \cdot \frac{e}{c} A(x) a_{0}=0, \\
-h b_{0 t}(x, t)+h \omega \cdot b_{0 x}-i \omega \cdot \frac{e}{c} A(x) b_{0}=0
\end{array}
$$

$$
\begin{aligned}
& h a_{p t}(x, t)+h \omega \cdot a_{p x}(x, t)-i \omega \cdot \frac{e}{c} A(x) a_{p}=i\left(\frac{h^{2}}{2 m} \frac{\partial^{2}}{\partial t^{2}}+H\right) a_{p-1} \\
& -h b_{p t}(x, t)+h \omega \cdot b_{p x}-i \omega \cdot \frac{e}{c} A(x) b_{p}=i\left(\frac{\partial^{2}}{\partial t^{2}}+H\right) b_{p-1}, \quad 1 \leq p \leq N
\end{aligned}
$$

We have $b_{p}(x, t)=a_{p}(x,-t)$ for $p \geq 0$, assuming that $b_{p}(x, 0)=a_{p}(x, 0)$.

Introduce new coordinates $(s, \tau, t)$ instead of $\left(x_{1}, x_{2}, t\right)$ where

$$
\begin{aligned}
& s=\left(x-x^{(0)}\right) \cdot \omega-t, \\
& \tau=\left(x-x^{(0)}\right) \cdot \omega_{\perp}, \\
& t=t .
\end{aligned}
$$


Here $\omega_{\perp} \cdot \omega=0,\left|\omega_{\perp}\right|=|\omega|=1$. We assume that $x^{(0)}$ is a fixed point outside of the obstacle $\Omega_{1}$ and that the line $x=x^{(0)}+s \omega, s \in \mathbf{R}$, does not intersect $\Omega_{1}$. Equations (3.8), (3.9) have the following form in the new coordinates

$$
\hat{a}_{0 t}(s, \tau, t)-i \omega \cdot \frac{e}{h c} A\left(x^{(0)}+(s+t) \omega+\tau \omega_{\perp}\right) \hat{a}_{0}=0,
$$

$$
\hat{a}_{p t}(s, \tau, t)-i \omega \cdot \frac{e}{h c} A\left(x^{(0)}+(s+t) \omega+\tau \omega_{\perp}\right) \hat{a}_{p}=\hat{f}_{p}(s, \tau, t), \quad p \geq 1,
$$

where $\hat{a}_{p}(s, \tau, t)=a_{p}(x, t), \hat{f}_{p}(s, \tau, t)$ is $\frac{i}{h}\left(\frac{h^{2}}{2 m} \frac{\partial^{2}}{\partial t^{2}}+H\right) a_{p-1}$ in the new coordinates.

We impose the following initial conditions

$$
\begin{array}{r}
\hat{a}_{0}(s, \tau, 0)=\frac{1}{2} \chi_{0}\left(\frac{\tau}{\delta_{1}}\right) \chi_{0}\left(\frac{s}{\delta_{2} k}\right), \\
\hat{a}_{p}(s, \tau, 0)=0 \text { for } p \geq 1,
\end{array}
$$

where $\chi_{0}(s)$ is the same as above. We assume that $\delta_{1}$ is such that supp $\chi_{0}\left(\frac{\left(x-x^{(0)}\right) \cdot \omega_{\perp}}{\delta_{1}}\right)$ does not intersect $\Omega_{1}$. Then

$\hat{a}_{0}(s, \tau, t)=\frac{1}{2} \chi_{0}\left(\frac{\tau}{\delta_{1}}\right) \chi_{0}\left(\frac{s}{\delta_{2} k}\right) \exp \left(\frac{i e}{h c} \int_{0}^{t} \omega \cdot A\left(x^{(0)}+\left(s+t^{\prime}\right) \omega+\tau \omega_{\perp}\right) d t^{\prime}\right)$.

Since $s=\left(x-x^{(0)}\right) \cdot \omega-t$ we have in the original coordinates

$$
\begin{aligned}
& a_{0}(x, t)=\frac{1}{2} \chi_{0}\left(\frac{\left(x-x^{(0)}\right) \cdot \omega_{\perp}}{\delta_{1}}\right) \chi_{0}\left(\frac{\left(x-x^{(0)}\right) \cdot \omega-t}{\delta_{2} k}\right) \\
& \cdot \exp \left(\frac{i e}{h c} \int_{0}^{t} \omega \cdot A\left(x-t^{\prime \prime} w\right) d t^{\prime \prime}\right)
\end{aligned}
$$

where we made the change of variables $t-t^{\prime}=t^{\prime \prime}$. Note that

$$
\left|a_{p}(x, t)\right| \leq C t^{p}, \quad 1 \leq p \leq N
$$

and (3.5) holds for any $r \geq 1$. Since $b_{p}(x, t)=a_{p}(x,-t), p \geq 0$, we have that

$$
\begin{array}{r}
w_{N}(x, 0)=\chi_{0}\left(\frac{\left(x-x^{(0)}\right) \cdot \omega_{\perp}}{\delta_{1}}\right) \chi_{0}\left(\frac{\left(x-x^{(0)}\right) \cdot \omega}{\delta_{2} k}\right) e^{i \frac{m}{h} k \omega \cdot x} \\
w_{N t}(x, 0)=0 .
\end{array}
$$


Let

$$
u_{N}(x, t)=\frac{e^{-i \frac{\pi}{4}} \sqrt{m}}{\sqrt{2 \pi h t}} \int_{-\infty}^{\infty} e^{\frac{i m x_{0}^{2}}{2 h t}} w_{N}\left(x, x_{0}\right) d x_{0} .
$$

Using that $b_{p}(x, t)=a_{p}(x,-t)$ and making a change of variables we get

$$
u_{N}(x, t)=\frac{2 e^{-i \frac{\pi}{4}} \sqrt{m}}{\sqrt{2 \pi h t}} \int_{-\infty}^{\infty} e^{\frac{i m x_{0}^{2}}{2 h t}+\frac{i m k}{h}\left(x \cdot \omega-x_{0}\right)} \sum_{p=0}^{N} \frac{a_{p}\left(x, x_{0}\right)}{(i k)^{p}} d x_{0} .
$$

We have

$$
\left(-i h \frac{\partial}{\partial t}+H\right) u_{N}(x, t)=\frac{e^{-i \frac{\pi}{4}} \sqrt{m}}{\sqrt{2 \pi h t}} \int_{-\infty}^{\infty} e^{\frac{i m x_{0}^{2}}{2 h t}}\left(\frac{h^{2}}{2 m} \frac{\partial^{2}}{\partial x_{0}^{2}}+H\right) w_{N}\left(x, x_{0}\right) d x_{0} .
$$

Note that

$$
\begin{aligned}
\left(\frac{h^{2}}{2 m} \frac{\partial^{2}}{\partial x_{0}^{2}}+H\right) w_{N}\left(x, x_{0}\right)=e^{\frac{i m k}{h}\left(x \cdot \omega-x_{0}\right)}\left(\frac{h^{2}}{2 m} \frac{\partial^{2}}{\partial x_{0}^{2}}+H\right) a_{N}\left(x, x_{0}\right) & \\
& +e^{\frac{i m k}{h}\left(x \cdot \omega+x_{0}\right)}\left(\frac{h^{2}}{2 m} \frac{\partial^{2}}{\partial x_{0}^{2}}+H\right) b_{N}\left(x, x_{0}\right) .
\end{aligned}
$$

Denote by $g_{N}(x, t)$ the right hand side of (3.18). Since $b_{N}\left(x, x_{0}\right)=a_{N}\left(x,-x_{0}\right)$ we have

$$
g_{N}(x, t)=\frac{2 e^{-i \frac{\pi}{4}} \sqrt{m}}{\sqrt{2 \pi h t}} \int_{-\infty}^{\infty} e^{\frac{i m x_{0}^{2}}{2 h t}} e^{\frac{i m k}{h}\left(x \cdot \omega-x_{0}\right)}\left(\frac{h^{2}}{2 m} \frac{\partial^{2}}{\partial x_{0}^{2}}+H\right) \frac{a_{N}\left(x, x_{0}\right)}{(i k)^{N}} d x_{0} .
$$

We apply the stationary phase method to the integral (3.17). The equation for the critical point is $\frac{m x_{0}}{h t}-\frac{m k}{h}=0$, i.e. $x_{0}=k t$ and the Hessian is $\frac{m}{h t}$. Therefore

$$
\begin{array}{r}
u_{N}(x, t)=e^{-\frac{i m k^{2} t}{2 h}+i \frac{m k}{h} x \cdot \omega} \chi_{0}\left(\frac{\left.\left(x-x^{(0)}\right) \cdot \omega_{\perp}\right)}{\delta_{1}}\right) \exp \left(\frac{i e}{h c} \int_{0}^{\infty} \omega \cdot A\left(x-s^{\prime} \omega\right) d s^{\prime}\right) \\
+O(\varepsilon),
\end{array}
$$

where $\varepsilon$ is arbitrary small when $k$ is sufficiently large and $t$ is sufficiently small. 
We used that $\chi_{0}\left(\frac{x \cdot \omega-k t}{\delta_{2} k}\right)=1$ when $k$ is large and $t$ is small. Note that

$$
\left|\frac{a_{p}(x, k t)}{(i k)^{p}}\right| \leq \frac{C}{k^{p}}(k t)^{p} \leq C t^{p}
$$

is small when $t$ is small.

Applying the stationary phase method to (3.19) we get, using (3.14), that

$$
\int_{\mathbf{R}^{2} \backslash \Omega}\left|g_{N}(x, t)\right|^{2} d x \leq C t^{2 N} k .
$$

We used in (3.22) that $\chi_{0}\left(\frac{x \cdot \omega-k t}{\delta_{2} k}\right)=0$ when $|x \cdot \omega|>C k$.

Denote by $\left\|g_{N}\right\|_{r}$ the Sobolev norm in $H_{r}\left(\mathbf{R}^{2} \backslash \Omega_{1}\right)$. It follows from (3.19) and (3.14) that

$$
\left\|g_{N}\right\|_{r} \leq C t^{N} k^{r+\frac{1}{2}}
$$

Let $R_{N}(x, t)$ be the solution of

$$
\begin{gathered}
\left(-i h \frac{\partial}{\partial t}+H\right) R_{N}=-g_{N}(x, t) \text { in }\left(\mathbf{R}^{2} \backslash \Omega_{1}\right) \times(0, T), \\
\left.R_{N}\right|_{\partial \Omega_{1} \times(0, T)}=0, \\
R_{N}(x, 0)=0 .
\end{gathered}
$$

Such solution exists and satisfies the following estimates (cf. [E1]):

$$
\max _{0 \leq t \leq T}\left\|R_{N}(\cdot, t)\right\|_{2} \leq C \int_{0}^{T}\left(\left\|g_{N}(\cdot, t)\right\|_{0}+\left\|\frac{\partial g_{N}(\cdot, t)}{\partial t}\right\|_{0}\right) d t .
$$

By the Sobolev embedding theorem $\left|R_{N}(x, t)\right| \leq C \max _{0 \leq t \leq T}\left\|R_{N}(\cdot, t)\right\|_{2}$ for all $(x, t) \in\left(\mathbf{R}^{2} \backslash \Omega_{1}\right) \times(0, T)$. Since

$$
\max _{0 \leq t \leq T}\left\|\frac{\partial^{p}}{\partial t^{p}} g_{N}(x, t)\right\|_{r} \leq C T^{N} k^{\frac{1}{2}+r}
$$

we get that

$$
\left|R_{N}(x, t)\right| \leq C \varepsilon
$$

if $T \leq \frac{C}{k^{\delta_{3}}}, 0<\delta_{3}<1,(N+1) \delta_{3}>\frac{1}{2}, k$ is large. 
Note that $u=u_{N}+R_{N}$ satisfies (1.1), (1.2) and the initial condition $u(x, 0)=u_{N}(x, 0)=\chi_{0}\left(\frac{\left(x-x^{(0)}\right) \cdot \omega_{\perp}}{\delta_{1}}\right) \chi_{0}\left(\frac{\left(x-x^{(0)}\right) \cdot \omega}{\delta_{2} k}\right) e^{i \frac{m k}{h} x \cdot \omega}$. Therefore we constructed a solution $u(x, t, \omega)$ for $x \in \mathbf{R}^{2} \backslash \Omega_{1}, t \in(0, T), T=O\left(\frac{1}{k_{3}^{\delta}}\right), k$ is large, such that

$$
=e^{-i \frac{m k^{2} t}{2 h}-i \frac{m k}{h} x \cdot \omega} \chi_{0}\left(\frac{\left(x-x^{(0)}\right) \cdot \omega_{\perp}}{\delta_{1}}\right) \exp \left(i \frac{e}{h c} \int_{0}^{\infty} \omega \cdot A\left(x-s^{\prime} \omega\right) d s^{\prime}\right)+O(\varepsilon),
$$

where $\varepsilon$ can be chosen arbitrary small if $k$ is large enough. Note that the integral in (3.24) converges since $A(x)=C \frac{\left(x_{2},-x_{1}\right)}{|x|^{2}}+O\left(\frac{1}{|x|^{2}}\right)$.

Let $x^{(0)} \in \mathbf{R}^{2} \backslash \Omega_{1}$ and let $\omega$ and $\theta$ be two unit vectors (see Fig.1):

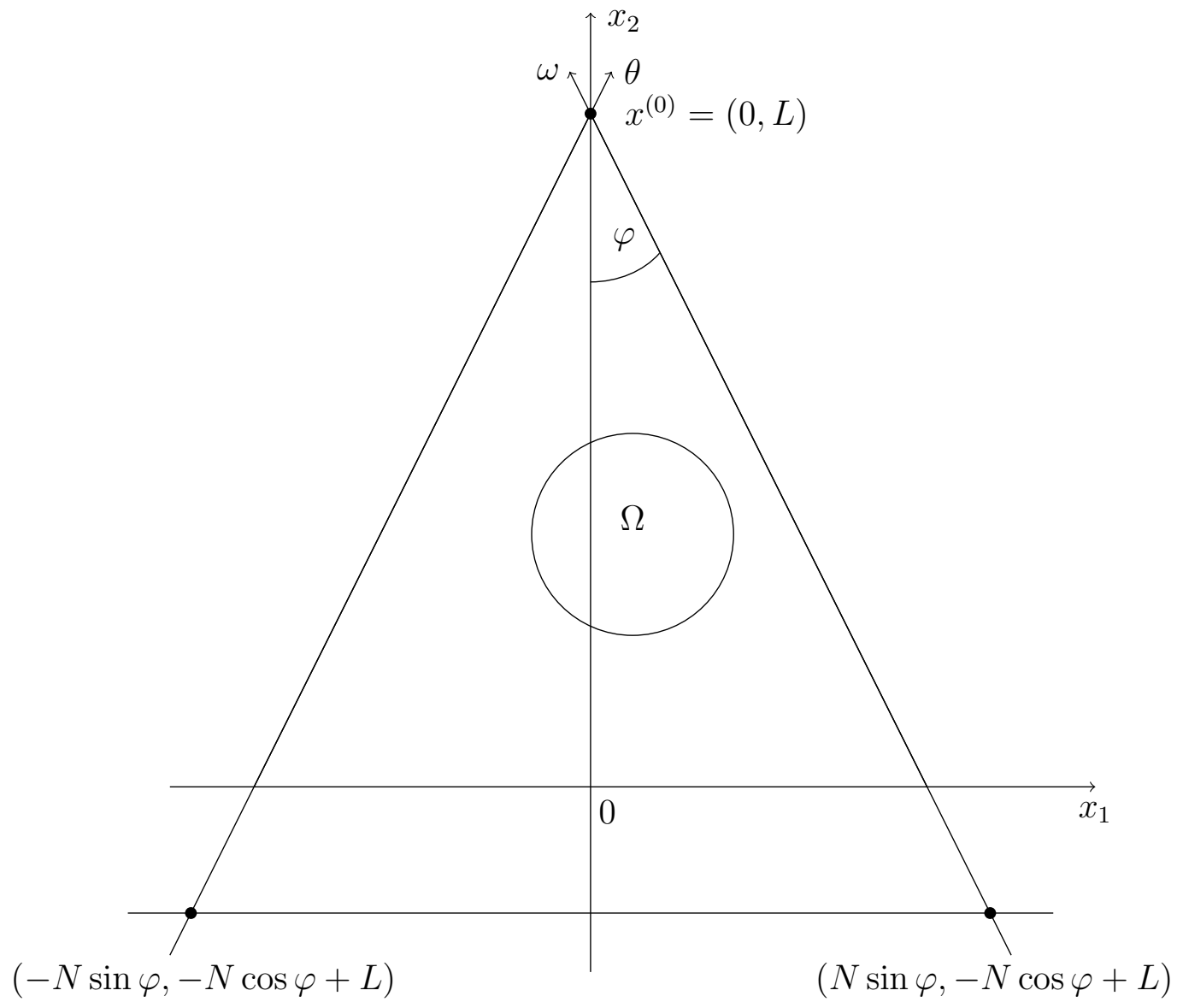

Figure 1. 
Consider the difference of two solutions of the form (3.24) corresponding to $\left(x^{(0)}, \omega\right)$ and $\left(x^{(0)}, \theta\right)$, respectively:

$$
w(x, t)=u(x, t, \omega)-u(x, t, \theta),
$$

where $u(x, t, \omega)$ is the same as in (3.24) and

(3.26) $u(x, t, \theta)$

$$
=e^{-i \frac{m k^{2} t}{2 h}+\frac{i m k}{h} x \cdot \theta} \chi_{0}\left(\frac{\left(x-x^{(0)}\right) \cdot \theta_{\perp}}{\delta_{1}}\right) \exp \left(i \frac{e}{h c} \int_{0}^{\infty} \theta \cdot A\left(x-s^{\prime} \theta\right) d s^{\prime}\right)+O(\varepsilon),
$$

where $\theta_{\perp} \cdot \theta=0$. Note that modulo $O(\varepsilon)$ the support of $v_{1}$ is contained in a small neighborhood of the line $x=x^{(0)}+s \omega$ and the support of $v_{2}$ is contained in a small neighborhood of $x=x^{(0)}+s \theta$.

Let $U_{0}$ be a disk of radius $\varepsilon_{0}$ centered in $x^{(0)}$ and contained in $\left(\operatorname{supp} v_{1}\right) \cap$ $\left(\operatorname{supp} v_{2}\right)$. We assume that $\chi_{0}\left(\frac{\left(x-x^{(0)}\right) \cdot \omega_{\perp}}{\delta_{1}}\right)=\chi_{0}\left(\frac{\left(x-x^{(0)}\right) \cdot \theta_{\perp}}{\delta_{1}}\right)=1$ in $U_{0}$. We have for $x \in U_{0}$ and $0<t<T=\frac{1}{k^{\delta_{3}}}$

$$
\begin{aligned}
& \mid u(x, t, \omega)-\left.u(x, t, \theta)\right|^{2}=\left|1-e^{i \frac{m k}{h} x \cdot(\omega-\theta)+i\left(I_{1}-I_{2}\right)}\right|^{2}+O(\varepsilon) \\
&= 4 \sin ^{2} \frac{1}{2}\left(\frac{m k}{h} x \cdot(\omega-\theta)+I_{1}-I_{2}\right)+O(\varepsilon) \\
& I_{1}=\frac{e}{h c} \int_{0}^{\infty} \omega \cdot A(x-s \omega) d s, \quad I_{2}=\frac{e}{h c} \int_{0}^{\infty} \theta \cdot A(x-s \theta) d s
\end{aligned}
$$

and $k>k_{0}, k_{0}$ is large, $T \leq \frac{1}{k_{0}^{\delta_{3}}}$.

Choose $k_{n}>k_{0}$ such that

$$
\frac{m k_{n}}{h} x^{(0)} \cdot(\omega-\theta)=2 \pi n, \quad n \in \mathbf{Z}
$$

Let, for simplicity, $\theta_{1}=-\omega_{1}, \theta_{2}=\omega_{2}, x^{(0)}=(0, L), \tan \varphi=\frac{\theta_{1}}{\theta_{2}}$ is small. Define

$$
\begin{aligned}
I_{1 N}(x, \omega) & =\frac{e}{h c} \int_{0}^{N} \omega \cdot A(x-s \omega) d s, \\
I_{2 N}(x, \theta) & =\frac{e}{h c} \int_{0}^{N} \theta \cdot A(x-s \theta) d s,
\end{aligned}
$$




$$
I_{3 N}=\frac{e}{h c} \int_{-N \sin \varphi}^{N \sin \varphi} A_{1}(s,-N \cos \varphi+L) d s
$$

(see Fig.1). Note that

$$
-I_{1 N}\left(x^{(0)}, \omega\right)+I_{2 N}\left(x^{(0)}, \theta\right)+I_{3 N}=\alpha,
$$

where $\alpha$ is the magnetic flux (cf. (1.4)). We assume that

$$
\alpha \neq 2 \pi n, \forall n \in \mathbf{Z} \text {. }
$$

Since $|A| \leq \frac{C}{r}$, where $r$ is the distance to $\Omega_{1}$, we have

$$
\left|I_{3 N}\right| \leq \frac{e}{h c} \frac{C}{N} 2 N \sin \varphi=C_{1} \frac{e}{h c} \sin \varphi
$$

When $N \rightarrow \infty$ we get

$$
I_{2}-I_{1}=\alpha+O\left(\frac{e}{h c} \sin \varphi\right) \text { for } x \in U_{0} .
$$

Assuming that the radius of the disk $U_{0}$ is $\varepsilon_{0}$ we get

$$
\left|\frac{m k_{n}}{h}\left(x-x^{(0)}\right) \cdot(\omega-\theta)\right| \leq 2 \frac{m k_{n}}{h} \varepsilon_{0} \sin \varphi .
$$

Therefore for arbitrary small $\varepsilon>0$ using (3.28), (3.29), (3.31), (3.32), (3.33), fixing $k_{n}>k_{0}$ and choosing $\varphi$ and $\varepsilon_{0}$ small enough we get

$$
|u(x, t, \omega)-u(x, t, \theta)|^{2}=4 \sin ^{2} \frac{\alpha}{2}+O(\varepsilon),
$$

where $x \in U_{0}$ and $\alpha$ is the magnetic flux (1.4).

Therefore, Theorem 1.1 is proven.

\subsection{The three-dimensional case}

The constructions of the subsection 3.1 can be carried out in the case of three dimensions. Consider, for example, a toroid $\Omega_{1}$ in $\mathbf{R}^{3}$ as in Tonomura et al experiment (cf [ $\mathrm{T}$ et al]). Let $x^{(0)}$ be a point outside of $\Omega_{1}$ and let $\gamma_{1}=\left\{x=x^{(0)}+s \omega, s \leq 0\right\}$ be a ray passing through the hole of the toroid. As in subsection 3.1 we can construct a solution $v_{1}(x, t, \omega)$ of the form (3.26). In the case $n \geq 3$ dimensions there are $(n-1)$ orthogonal 
unit vectors $\omega_{\perp j}, 1 \leq j \leq n-1$, such that $\omega \cdot \omega_{\perp j}=0,1 \leq j \leq n-1$, and we have to replace $\chi_{0}\left(\frac{\left(x-x^{(0)}\right) \cdot \omega_{\perp}}{\delta_{1}}\right)$ in $(\underline{3.26})$ by $\prod_{j=1}^{n-1} \chi_{0}\left(\frac{\left(x-x^{(0)}\right) \cdot \omega_{\perp j}}{\delta_{1}}\right)$. Let $\gamma_{2}=\left\{x=x^{(0)}+s \theta, s \leq 0\right\}$ be a ray passing outside of toroid and let $v_{2}(x, t, \omega)$ be the corresponding solution of the form (3.27). As in subsection 3.1 we get

$$
\left|v_{1}(x, t, \omega)-v_{2}(x, t, \theta)\right|^{2}=4 \sin ^{2} \frac{\alpha}{2}+O(\varepsilon),
$$

where $\alpha=\frac{e}{h c} \int_{\gamma} A(x) \cdot d x, \gamma$ is a closed simple curve encircling $\Omega_{1}$ and we assume that the angle between $\omega$ and $\theta$ is small.

Assuming that $\alpha \neq 2 \pi n, \forall n \in \mathbf{Z}$, we obtain that the probability density $\left|v_{1}-v_{2}\right|^{2}$ depends on $\alpha$ and this proves the AB effect.

\subsection{The case of several obstacles}

Let $\Omega_{j}, 1 \leq j \leq m, m>1$, be obstacles in $\mathbf{R}^{2}$, and let $\alpha_{j}=\frac{e}{h c} \int_{\gamma_{j}} A(x) \cdot d x$ be the magnetic fluxes generated by magnetic fields shielded in $\Omega_{j}, 1 \leq j \leq m$. Suppose that some $\alpha_{j}$ satisfy the condition (3.30). If the obstacles are close to each other it is impossible to repeat the construction of the subsection 3.1 separately for each $\Omega_{j}$. Note that if the total flux $\sum_{j=1}^{m} \alpha_{j}=2 \pi p, p \in \mathbf{Z}$, then treating $\Omega=\cup_{j=1}^{m} \Omega_{j}$ as one obstacle we will miss the magnetic AB effect.

We shall introduce some notations.

Let $x^{(1)} \notin \Omega=\cup_{j=1}^{m} \Omega_{j}$. Denote by $\gamma=\gamma_{1} \cup \gamma_{2} \cup \ldots \cup \gamma_{r}$ the broken ray starting at $x^{(1)}$ and reflecting at $\Omega$ at points $x^{(2)}, \ldots, x^{(r)}$. The last leg $\gamma_{r}$ can be extended to the infinity. Denote by $\omega_{p}, 1 \leq p \leq r$, the directions of $\gamma_{p}$. The equations of $\gamma_{1}, \ldots, \gamma_{r}$ are $x=x^{(1)}+s \omega_{1}, s_{1}=0 \leq s \leq s_{2}, x=$ $x^{(2)}+s \omega_{2}, s_{2} \leq s \leq s_{3}, \ldots, x=x^{(r)}+s \omega_{r}, s_{r} \leq s<+\infty$. Here $s_{p}$ are such that $x\left(s_{p}\right)=x^{(p)}, 1 \leq p \leq r$. Denote by $\tilde{\gamma}=\tilde{\gamma}_{1} \cup \tilde{\gamma}_{2} \cup \ldots \cup \tilde{\gamma}_{r}$ the lifting of $\gamma$ to $\mathbf{R}^{2} \times(0,+\infty)$, where the equations of $\tilde{\gamma}_{p}$ are $x=x^{(p)}+s \omega_{p}, t=s, s_{p} \leq$ $s \leq s_{p+1}, s_{r+1}=+\infty$. Note that the times when $\tilde{\gamma}$ hits the obstacles are $t_{p}=s_{p}, 2 \leq p \leq r$.

Let $V_{0}$ be a small neighborhood of $x^{(1)}$. Denote by $\gamma_{y}=\cup_{p=1}^{r} \gamma_{p y}$ the broken ray that starts at $y \in V_{0}$. We assume that $\gamma_{1 y}$ has the form $x=$ $y+s \omega_{1}, 0 \leq s \leq s_{2}(y)$, where $x^{(2)}(y)=y+s_{2}(y) \omega_{1}$ is the point where $\gamma_{1 y}$ hits $\partial \Omega$. We have $\gamma_{x^{(1)}}=\gamma$. Let $U_{0}(t)=\{x=x(t)\}$ be the set of endpoints at the time $t$ of $\tilde{\gamma}_{r y}, y \in V_{0}$. Note that there is a one-to-one correspondence between $y \in V_{0}$ and $x(t) \in U_{0}(t)$. Therefore we shall denote the broken ray 
starting at $y \in V_{0}$ and ending at $x(t)$ at the time $t$ by $\gamma(x(t))$ instead of $\gamma_{y}$. As in [E6] we can construct a geometric optics solution of $\left(\frac{h^{2}}{2 m} \frac{\partial^{2}}{\partial t^{2}}+H\right) w_{N}=0$ in $\left(\mathbf{R}^{2} \backslash \Omega\right) \times(0,+\infty)$ in the form

$$
\begin{aligned}
& w_{N}(x, t) \\
& =\sum_{p=1}^{r} \sum_{n=0}^{N} e^{i \frac{m k}{h}\left(\psi_{p}(x)-t\right)} \frac{a_{p n}(x, t)}{(i k)^{n}}+\sum_{p=1}^{r} \sum_{n=0}^{N} e^{i \frac{m k}{h}\left(\psi_{p}(x)+t\right)} \frac{b_{p n}(x, t)}{(i k)^{n}}
\end{aligned}
$$

where

$$
\begin{gathered}
\left|\nabla \psi_{p}(x)\right|=1, \quad \frac{\partial \psi_{p}\left(x^{(p)}\right)}{\partial x}=\omega_{p}, \quad 1 \leq p \leq r \\
\psi_{1}(x)=x \cdot \omega_{1} .
\end{gathered}
$$

We have that $a_{p n}(x, t)=b_{p n}(x,-t)$ and $a_{p n}(x, t)$ satisfy the transport equations

$$
\begin{gathered}
\frac{\partial a_{p n}}{\partial t}+\frac{\partial \psi_{p}(x)}{\partial x} \cdot \frac{\partial a_{p n}}{\partial x}+\frac{1}{2} \Delta \psi_{p} a_{p n}-i \frac{e}{h c} A(x) \cdot \frac{\partial \psi_{p}}{\partial x} a_{p n}=f_{p n}(x, t) \\
1 \leq p \leq r, 0 \leq n \leq N
\end{gathered}
$$

where $f_{p 0}=0, f_{p n}$ depend on $a_{p j}$ for $n \geq 1,0 \leq j \leq n-1$. The following boundary conditions hold on $\partial \Omega \times(0,+\infty)$ :

$$
\begin{aligned}
& \left.\psi_{p}\right|_{\partial \Omega \times(0,+\infty)}=\left.\psi_{p+1}\right|_{\partial \Omega \times(0,+\infty)}, \quad 1 \leq p \leq r-1, \\
& \left.a_{p n}\right|_{\partial \Omega \times(0,+\infty)}=-\left.a_{p+1, n}\right|_{\partial \Omega \times(0,+\infty)}, \quad 1 \leq p \leq r-1 .
\end{aligned}
$$

Conditions (3.38) imply that

$$
\left.w_{N}\right|_{\partial \Omega \times(0,+\infty)}=0 .
$$

We impose the following initial conditions:

$$
\begin{aligned}
& a_{10}(x, 0)=\frac{1}{2} \chi_{0}\left(\frac{\left(x-x^{(1)}\right) \cdot \omega_{1 \perp}}{\delta_{1}}\right) \chi_{0}\left(\frac{\left(x-x^{(1)}\right) \cdot \omega_{1}}{\delta_{2} k}\right), \\
& a_{1 n}(x, 0)=0, \quad n \geq 1 .
\end{aligned}
$$

We assume that $\delta_{1}, \delta_{2}$ in (3.37) are small, so that the support of the first sum in (3.35) is contained in a small neighborhood of $\tilde{\gamma}=\cup_{p=1}^{r} \tilde{\gamma}_{p}$. We define $a_{p n}(x, t)$ as zero outside of this neighborhood of $\tilde{\gamma}$. 
Let $x^{(0)} \in \gamma_{r}$ and $\left(x^{(0)}, t^{(0)}\right)$ be a corresponding point on $\tilde{\gamma}_{r}$. It was shown in $[\mathrm{E} 6]$ that

$$
a_{r 0}(x, t)=c_{0}(x, t) \exp \left(\frac{i e}{h c} \int_{\tilde{\gamma}(x, t)} A(x) \cdot d x\right)+O\left(\frac{1}{k}\right),
$$

where $\tilde{\gamma}(x, t)$ is the broken ray starting in a neighborhood of $x^{(1)}$ at $t=0$ and ending at $(x, t), c(x, t) \neq 0$ in a neighborhood of $\left(x^{(0)}, t^{(0)}\right)$.

As in subsection 3.1 we have that $a_{r n}(x, t), n \geq 1$ satisfy the estimates of the form 3.5$)$.

Let $\tilde{\gamma}\left(x^{(0)}, t^{(0)}\right)$ be the broken ray starting at $\left(x^{(1)}, 0\right)$ and ending at $\left(x^{(0)}, t^{(0)}\right)$, where $x^{(0)} \in \gamma_{r}$. Let

$$
u_{N}(x, t)=\frac{e^{-i \frac{\pi}{4}} \sqrt{m}}{\sqrt{2 \pi h t}} \int_{-\infty}^{\infty} e^{\frac{i m x_{0}^{2}}{2 h t}} w_{N}\left(x, x_{0}\right) d x_{0}
$$

where $w_{N}\left(x, x_{0}\right)$ is the same as in (3.35). We assume in this subsection that

$$
t=\frac{t^{\prime}}{k}, \quad 0 \leq t^{\prime} \leq T^{\prime}
$$

Applying the stationary phase method to (3.41) and using (3.40), (3.42) we get for $x$ belonging to a neighborhood of $x^{(0)}$

$$
\begin{array}{r}
u_{N}(x, t) \\
=\exp \left(i\left(-\frac{m k^{2} t}{2 h}+\frac{m k}{h} \psi_{r}(x)\right)\right) c_{0}\left(x, t^{\prime}\right) \exp \left(\frac{i e}{h c} \int_{\tilde{\gamma}\left(x, t^{\prime}\right)} A(x) \cdot d x\right) \\
+O\left(\frac{1}{k}\right)
\end{array}
$$

where $t^{\prime}=k t, t^{\prime}$ belongs to a neighborhood of $t^{(0)}$.

Since $A(x)$ is independent of $t$ we have

$$
\int_{\tilde{\gamma}\left(x, t^{\prime}\right)} A(x) \cdot d x=\int_{\gamma\left(x\left(t^{\prime}\right)\right)} A(x) \cdot d x
$$

where $\gamma\left(x\left(t^{\prime}\right)\right)$ is the projection of $\tilde{\gamma}\left(x, t^{\prime}\right)$ on the $x$-plane.

Analogously to subsection 3.1 we get that there exists $R_{N}(x, t)$ such that $R_{N}(x, t)=O\left(\frac{1}{k}\right), t=\frac{t^{\prime}}{k}, 0 \leq t^{\prime} \leq T^{\prime}$, and

$$
u(x, t)=u_{N}(x, t)+R_{N}(x, t)
$$


is the exact solution of (1.1) with the boundary conditions $\left.u\right|_{\partial \Omega \times\left(0, \frac{T^{\prime}}{k}\right)}=0$ and the initial condition

$$
u(x, 0)=\chi_{0}\left(\frac{\left(x-x^{(1)}\right) \cdot \omega_{1 \perp}}{\delta_{1}}\right) \chi_{0}\left(\frac{\left(x-x^{(1)}\right) \cdot \omega_{1}}{\delta_{2} k}\right) e^{i \frac{m k}{h} x \cdot \omega} .
$$

Denote by $\tilde{\beta}=\left\{x=x^{(2)}+s \theta, t=s, 0 \leq s \leq t^{(0)}\right\}$ the ray starting at $\left(x^{(2)}, 0\right)$ and ending exactly at the point $\left(x^{(0)}, t^{(0)}\right)$ (see Fig. 2). Analogously to (3.27) we can construct a solution $v(x, t)$ of (1.1), satisfying (1.2) and such that

$$
\begin{aligned}
v(x, t) & =\chi_{0}\left(\frac{\left(x-x^{(2)}\right) \cdot \theta_{\perp}}{\delta_{1}}\right) \chi_{0}\left(\frac{\left(x-x^{(2)}\right) \cdot \theta-k t}{\delta_{2} k}\right) c_{1}\left(x, t^{\prime}\right) \\
& \cdot \exp \left(-i \frac{m k^{2} t}{2 h}+i \frac{m k}{h} x \cdot \theta+\frac{i e}{h c} \int_{\tilde{\beta}\left(x, t^{\prime}\right)} A(x) \cdot d x\right)+O\left(\frac{1}{k}\right)
\end{aligned}
$$

where $t=\frac{t^{\prime}}{k},\left(x, t^{\prime}\right) \in U_{0}$, where $U_{0}$ is a neighborhood of $\left(x^{(0)}, t^{(0)}\right)$.

We choose initial conditions $c_{1}(x, 0)$ such that (cf. (3.43))

$$
c_{1}\left(x^{(0)}, t^{(0)}\right)=c\left(x^{(0)}, t^{(0)}\right) .
$$

Note that

$$
\int_{\tilde{\beta}\left(x, t^{\prime}\right)} A \cdot d x=\int_{\beta\left(x\left(t^{\prime}\right)\right)} A(x) \cdot d x,
$$

where $\beta\left(x\left(t^{\prime}\right)\right)$ is the projection of $\tilde{\beta}\left(x, t^{\prime}\right)$.

As in (3.28) we have for $(x, t)$ near $\left(x^{(0)}, \frac{t^{(0)}}{k}\right)$

$|u(x, t)-v(x, t)|^{2}=\left|c\left(x^{(0)}, t^{(0)}\right)\right|^{2} 4 \sin ^{2} \frac{1}{2}\left(\frac{m k}{h}\left(\psi_{r}(x)-\theta \cdot x\right)+I_{1}-I_{2}\right)+O(\varepsilon)$,

where

$$
I_{1}=\frac{e}{h c} \int_{\gamma\left(x^{(0)}\right)} A \cdot d x, \quad I_{2}=\frac{e}{h c} \int_{\beta\left(x^{(0)}\right)} A \cdot d x .
$$

Choose $k_{n}>k_{0}$ such that

$$
\frac{m k_{n}}{h}\left(\psi_{r}\left(x^{(0)}\right)-x^{(0)} \cdot \theta\right)=2 \pi n, n \in \mathbf{Z}
$$


and choose the initial points $x^{(1)}$ on $\gamma_{1}$ and $x^{(2)}$ on $\beta$ far enough from $\Omega$ to have the integral

$$
I_{3}=\frac{e}{h c} \int_{\sigma} A \cdot d x
$$

small. Here $\sigma$ is the straight line connecting $x^{(1)}$ and $x^{(2)}$ and not intersecting $\Omega$ (see Fig. 2). Then if the neighborhood $U_{0}$ is small enough we get

$$
|u(x, t)-v(x, t)|^{2}=\left|c\left(x^{(0)}, t^{(0)}\right)\right| 4 \sin ^{2} \frac{\alpha}{2}+O(\varepsilon),
$$

where $\alpha=I_{1}-I_{2}+I_{3}$ at $\left(x^{(0)}, t^{(0)}\right),(x, t)$ is close to $\left(x^{(0)}, \frac{t^{(0)}}{k}\right)$. Note that $\alpha$ is the sum of magnetic fluxes of obstacles that are bounded by $\gamma \cup(-\beta) \cup \sigma$.

We shall assume that this $\alpha \neq 0$ (modulo $p, p \in Z$ ). Analogously to Theorem 1.1 we get from (3.47) the following result.

Theorem 3.1. There exists a solution $u(x, t)$ of (1.1), (1.2) with highly oscillating initial data such that (3.47) holds in a neighborhood of $x^{(0)}=x\left(t^{(0)}\right)$. It follows from (3.47) that the magnetic potential affects the probability density $|u(x, t)|^{2}$ near $x^{(0)}=x\left(t^{(0)}\right)$ : the change in $\alpha$ leads to the change in $|u(x, t)|^{2}$. This proves the Aharonov-Bohm effect in the case of several obstacles.

Varying appropriately the broken rays we can recover all $\alpha_{j}(\bmod 2 \pi p)$, $1 \leq j \leq m$, up to a sign. 


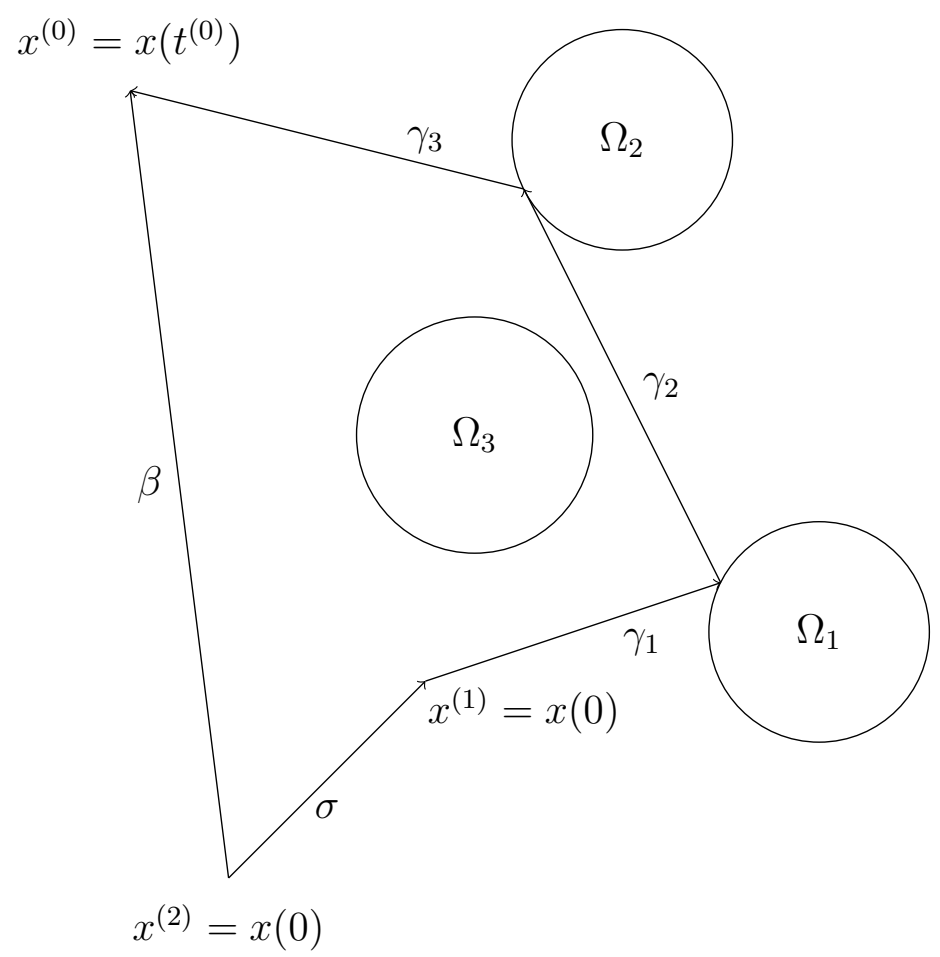

Figure 2.

Remark 3.1. The broken rays are useful also in the case of one obstacle. Consider two rays $\gamma_{1}$ and $\gamma_{2}$ starting at the same point $P_{0}$ reflecting from the artificial boundaries (mirrors) $M_{1}$ and $M_{2}$ and merging at some point $P_{1}$ behind the obstacle $\Omega$ (see Fig. 3). 


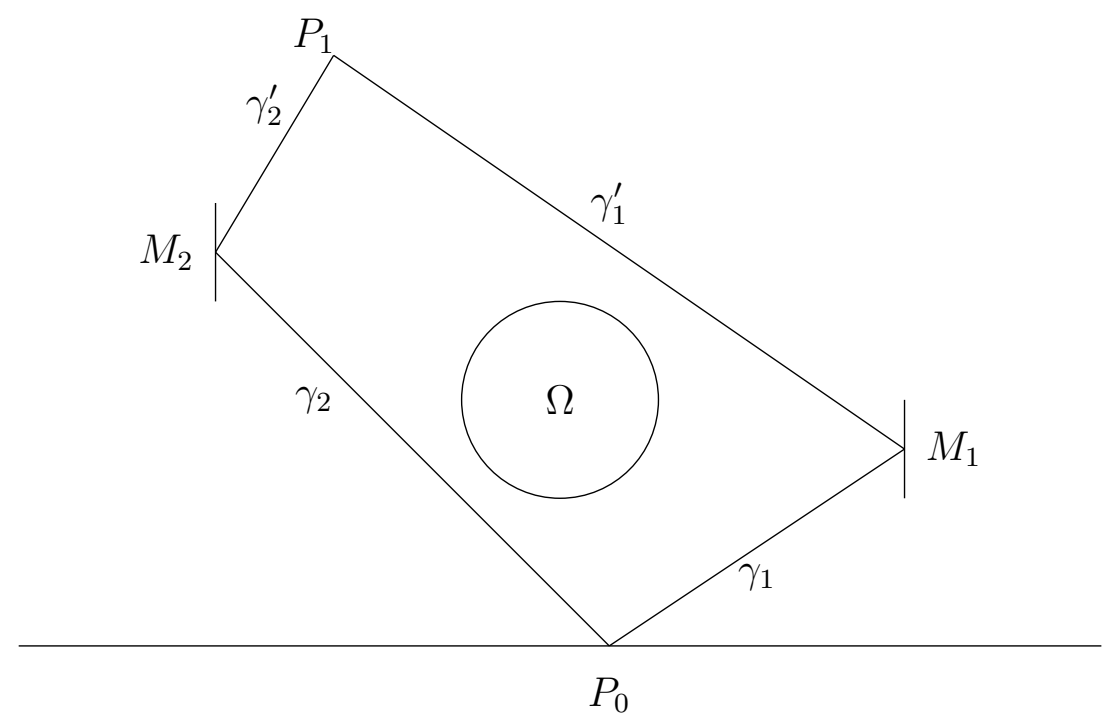

Figure 3.

One can construct solutions corresponding to the broken rays $\gamma_{1} \cup \gamma_{1}^{\prime}$ and $\gamma_{2} \cup \gamma_{2}^{\prime}$ and prove the magnetic AB effect. Note that this proof mimics the classical AB experiment where the beam of electrons is splitted at $P_{0}$ into two beams. Each of these beams reflects at $M_{1}$ and $M_{2}$ respectively and they merge at the interferometer at $P_{1}$.

In [BW1], [BW2] Ballesteros and Weder study the mathematical justification of the Tonomara and al. experiment by considering high frequency solutions of the Schrödinger equations concentrating along one straight ray. The use of broken rays may help to complete their results by taking into account the splitting, reflecting and merging of rays.

\section{The proof of the electric Aharonov-Bohm effect}

Let $D$ be a domain in $\mathbf{R}^{n} \times[0, T]$ and let $D_{t_{0}}=D \cap\left\{t=t_{0}\right\}$. Assume that $D_{t_{0}}$ depends continuously on $t_{0} \in[0, T]$ and that normals to $D \backslash\left(\overline{D_{0}} \cup \overline{D_{T}}\right)$ are not parallel to the $t$-axis. Suppose that the magnetic potential $A(x, t)=0$ in $D$ and consider the Schrödinger equation:

$$
i h \frac{\partial u(x, t)}{\partial t}+\frac{h^{2}}{2 m} \Delta u(x, t)-e V(x, t) u(x, t)=0, \quad(x, t) \in D,
$$


with zero Dirichlet boundary condition

$$
\left.u\right|_{\partial D_{t}}=0 \text { for } 0<t<T
$$

and nonzero initial condition

$$
u(x, 0)=u_{0}(x), \quad x \in D_{0} .
$$

Suppose that electric field $E=\frac{\partial V}{\partial x}=0$ in $D$. If $D_{t}$ are connected for all $t \in[0, T]$ then $V(x, t)=V(t)$, i.e. $V(t)$ does not depend on $x$. Making the gauge transformation

$$
v(x, t)=\exp \left(i \frac{e}{h} \int_{0}^{t} V\left(t^{\prime}\right) d t^{\prime}\right) u(x, t)
$$

we get that $v(x, t)$ satisfies the Schrödinger equation

$$
i h \frac{\partial v}{\partial t}+\frac{h^{2}}{2 m} \Delta v(x, t)=0
$$

where

$$
\begin{gathered}
\left.v\right|_{\partial D_{t}}=0 \text { for } 0<t<T, \\
v(x, 0)=u_{0}(x), x \in D_{0} .
\end{gathered}
$$

Therefore $V(t)$ is gauge equivalent to zero electric potential, i.e. there is no electric $\mathrm{AB}$ effect in the case when $D_{t_{0}}$ are connected for all $t_{0} \in[0, T]$. To have the electric $\mathrm{AB}$ effect the domain $D$ must be not connected on some subintervals of $(0, T)$.

We shall describe an electric AB effect when $A=0, E=0$ in $D$ but the electric potential $V(x, t)$ is not gauge equivalent to the zero potential.

Denote by $\Omega(\tau)$ the interior of the unit disk $x_{1}^{2}+x_{2}^{2} \leq 1$ with removed two parts $\Delta(\tau)$ and $\Delta(-\tau)$ depending on the parameter $\tau, 0 \leq \tau \leq \frac{1}{2}$ (see Fig. 4). 


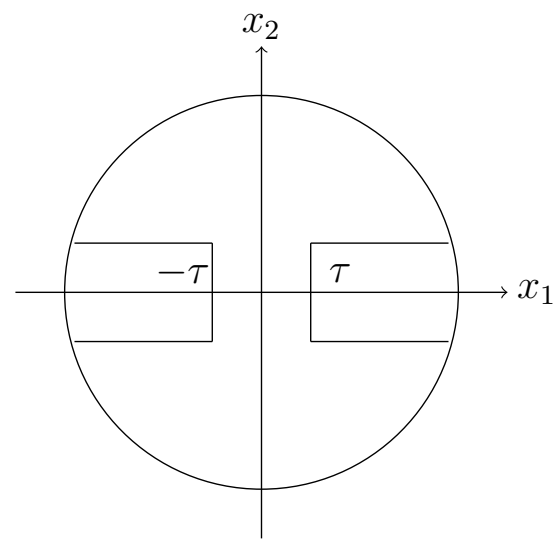

Figure 4.

Let $D$ be the following domain in $\mathbf{R}^{2} \times[0, T+1]$ :

$$
\begin{gathered}
D_{t}=\Omega\left(\frac{1}{2}-t\right) \text { for } 0 \leq t \leq \frac{1}{2}, \\
D_{t}=\Omega(0) \text { for } \frac{1}{2} \leq t \leq T+\frac{1}{2}, \\
D_{t}=\Omega\left(t-\frac{1}{2}-T\right) \text { for } T+\frac{1}{2} \leq t \leq T+1 .
\end{gathered}
$$

Here $D_{t_{0}}=D \cap\left\{t=t_{0}\right\}$.

Therefore $\Delta(\tau)$ and $\Delta(-\tau)$ increase in size from $\tau=\frac{1}{2}$ to $\tau=0$ when $0 \leq t \leq \frac{1}{2}$. Then they do not move for $\frac{1}{2} \leq t \leq T+\frac{1}{2}$. Note that $D_{t}$ consists of the components $D_{t}^{+}$and $D_{t}^{-}$for $\frac{1}{2} \leq t \leq \frac{1}{2}+T$, where $x_{2}>0$ in $D_{t}^{+}, x_{2}<0$ in $D_{t}^{-}$. When $T+\frac{1}{2} \leq t \leq T+1$ the parts $\Delta(\tau)$ and $\Delta(-\tau)$ return back to the initial position $\tau=\frac{1}{2}$.

Such moving domain is easy to realize. We can arrange that $V(x, t)=0$ in $D$ for $0 \leq t \leq \frac{1}{2}+\varepsilon$ and for $\frac{1}{2}+T-\varepsilon<t \leq T+1, V(x, t)=V_{1}(t)$ in $D_{t}^{+}, V(x, t)=V_{2}(t)$ in $D_{t}^{-}$for $\frac{1}{2} \leq t \leq T+\frac{1}{2}$. Then $E=\frac{\partial V(x, t)}{\partial x}=0$ in $D$.

We consider the Schrödinger equation (4.1) in $D$ with nonzero initial condition (4.3) and the zero boundary condition (4.2).

The gauge group $G(\bar{D})$ consists of all $g(x, t)$ in $\bar{D}$ such that $|g(x, t)|=1$. It follows from the topology of $D$ that any $g(x, t) \in G(\bar{D})$ has the form:

$$
g(x, t)=e^{i n \theta+\frac{i}{h} \varphi(x, t)},
$$


where $\varphi(x, t)$ is real-valued and differentiable in $\bar{D}$ and $\theta$ is the polar angle in $\left(x_{2}, t\right)$-plane. If $V_{1}(x, t)$ and $V_{2}(x, t)$ are gauge equivalent and if $\gamma$ is a closed contour in $D$ not homotopic to a point, then

$$
e \int_{\gamma} V_{1}(x, t) d t-e \int_{\gamma} V_{2}(x, t) d t=i h \int_{\gamma} g^{-1}(x, t) \frac{\partial g}{\partial t} d t=2 \pi h n, \quad n \in \mathbf{Z} .
$$

Let $\alpha_{j}=\frac{e}{h} \int_{\frac{1}{2}}^{T+\frac{1}{2}} V_{j}(t) d t, j=1,2$, and suppose $\alpha_{1}-\alpha_{2} \neq 2 \pi n, \forall n \in \mathbf{Z}$. Since the electric flux $\alpha=\frac{e}{h} \int_{\gamma} V(x, t) d t=\alpha_{1}-\alpha_{2} \neq 2 \pi n, \forall n$ the electric potential $V(x, t)$ is not gauge equivalent to the zero potential.

Now we are ready to prove Theorem 1.2 .

Proof of Theorem 1.2. Since $u(x, t)$ and $v(x, t)$ have the same initial and boundary conditions (4.2), (4.3) and $V(x, t)=0$ for $t \in\left[0, \frac{1}{2}\right]$ we have that $u(x, t)=v(x, t)$ for $t \in\left[0, \frac{1}{2}\right]$. Denote by $\Pi_{1}$ and $\Pi_{2}$ two connected components of $D \cap\left(\frac{1}{2}, T+\frac{1}{2}\right), \quad \Pi_{1} \quad=\quad \cup_{\frac{1}{2} \leq t \leq T+\frac{1}{2}} D_{t}^{+}$, $\Pi_{2}=\cup_{\frac{1}{2} \leq t \leq T+\frac{1}{2}} D_{t}^{-}$. Note that in $\Pi_{i}, i=1,2$, we have

$$
u(x, t)=v(x, t) \exp \left(-i \frac{e}{h} \int_{\frac{1}{2}}^{t} V_{i}\left(t^{\prime}\right) d t^{\prime}\right), \quad(x, t) \in \Pi_{i} .
$$

Let $c(x, t)=1$ for $t \leq \frac{1}{2}, c(x, t)=e^{-i \frac{e}{h} \int_{\frac{1}{2}}^{t} V_{i}\left(t^{\prime}\right) d t^{\prime}}$ in $\Pi_{i}$ for $t \in\left(\frac{1}{2}, T+\frac{1}{2}\right), i=$ 1,2 . Then $u(x, t)=c(x, t) v(x, t)$, i.e. $V(x, t)$ is gauge equivalent to zero in $D \cap\left(0, T+\frac{1}{2}\right)$.

Note that $u(x, t)$ and $v(x, t)$ satisfy the same equation (4.5) in $D \cap\left\{T+\frac{1}{2} \leq t \leq T+1\right\}$ and

$$
\begin{aligned}
& u\left(x, T+\frac{1}{2}\right)=e^{-i \alpha_{1}} v\left(x, T+\frac{1}{2}\right) \text { in } D_{T+\frac{1}{2}}^{+}, \\
& u\left(x, T+\frac{1}{2}\right)=e^{-i \alpha_{2}} v\left(x, T+\frac{1}{2}\right) \text { in } D_{T+\frac{1}{2}}^{-} .
\end{aligned}
$$

We shall show that the probability densities $|u(x, t)|^{2}$ and $|v(x, t)|^{2}$ are not equal identically for $T+\frac{1}{2}<t<T+\frac{1}{2}+\varepsilon$.

To prove that $|u(x, t)|^{2} \not \equiv|v(x, t)|^{2}$ we consider $w(x, t)=e^{i \alpha_{2}} u(x, t)$. Then $w\left(x, T+\frac{1}{2}\right)=v\left(x, T+\frac{1}{2}\right)$ in $D_{T+\frac{1}{2}}^{-}, w\left(x, T+\frac{1}{2}\right)=e^{i\left(\alpha_{2}-\alpha_{1}\right)} v\left(x, T+\frac{1}{2}\right)$ in $D_{T+\frac{1}{2}}^{+}$.

Proposition 4.1. Let $\left(0, x_{2}^{(0)}, T+\frac{1}{2}\right)$ be a point in $D_{T+\frac{1}{2}}^{-}$such that $v\left(0, x_{2}^{(0)}, T+\frac{1}{2}\right) \neq 0$. Let $O$ be a small neighborhood of $\left(0, x_{2}^{(0)}, T+\frac{1}{2}\right)$ in $D$. 
Suppose $v\left(x, T+\frac{1}{2}\right)=w\left(x, T+\frac{1}{2}\right)$ in $O \cap\left\{t=T+\frac{1}{2}\right\}$. If $|v(x, t)|^{2}=|w(x, t)|^{2}$ in $O \cap\left\{t>T+\frac{1}{2}\right\}$ then $v(x, t)=w(x, t)$ in $D \cap\left\{T+\frac{1}{2}<t<T+\frac{1}{2}+\varepsilon\right\}$.

Proof of Proposition 4.1. Let $R(x, t)=|v(x, t)|, \Phi(x, t)=\arg v(x, t)$, i.e. $v(x, t)=R(x, t) e^{i \Phi(x, t)}$. Substituting in (4.5) and separating the real and the imaginary parts we get

$$
\begin{gathered}
-h R_{t}=\frac{h^{2}}{2 m}(2 \nabla R \cdot \nabla \Phi+R \Delta \Phi), \\
h \Phi_{t} R=\frac{h^{2}}{2 m}\left(\Delta R-R|\nabla \Phi|^{2}\right) .
\end{gathered}
$$

Suppose $R$ is given. Then (4.9) is a first order partial differential equation in $\Phi$ and therefore the initial data $\Phi\left(x, T+\frac{1}{2}\right)$ in $O \cap\left\{t=T+\frac{1}{2}\right\}$ uniquely determines $\Phi(x, t)$ in the neighborhood $O$. Let $w=R_{1}(x, t) e^{i \Phi_{1}(x, t)}$. Note that $w(x, t)$ also satisfies (4.5) for $t>T+\frac{1}{2}$ and $\Phi_{1}$ satisfies (4.9) for $t>T+\frac{1}{2}$. Since $R=R_{1}$ in $O$ and, since $\Phi_{1}\left(x, T+\frac{1}{2}\right)=\Phi\left(x, T+\frac{1}{2}\right)$ in $O \cap\left\{t=T+\frac{1}{2}\right\}$, we have that $w(x, t)=v(x, t)$ in $O$. Then $w(x, t)=v(x, t)$ for $D \cap\left(T+\frac{1}{2}, T+\frac{1}{2}+\varepsilon\right)$ by the unique continuation property (see $[\mathrm{I}]$, section 6 ). By the continuity in $t$ we get $v\left(x, T+\frac{1}{2}\right)=w\left(x, T+\frac{1}{2}\right)$ in $D_{T+\frac{1}{2}}$ and this is a contradiction with $w\left(x, T+\frac{1}{2}\right)=e^{i\left(\alpha_{2}-\alpha_{1}\right)} v\left(x, T+\frac{1}{2}\right)$ in $D_{T+\frac{1}{2}}^{+}$, assuming that $v\left(x, T+\frac{1}{2}\right) \not \equiv 0$ in $D_{T+\frac{1}{2}}^{+}$.

Therefore $|u(x, t)|^{2} \not \equiv|v(x, t)|^{2}$ for $T+\frac{1}{2}<t<T+\frac{1}{2}+\varepsilon$, i.e. the AB effect holds.

Remark 4.1. In the proof of Proposition 4.1 we used that $R(x, t)=$ $|v(x, t)| \not \equiv 0$ in $D_{T+\frac{1}{2}}^{-}$and $|v(x, t)| \not \equiv 0$ in $D_{T+\frac{1}{2}}^{+}$. We shall show that this can be achieved by the appropriate choice of the initial condition $u_{0}(x)$ in (4.7). Choose any $v\left(x, T+\frac{1}{2}\right)$ such that $v\left(x, T+\frac{1}{2}\right) \not \equiv 0$ in $D_{T+\frac{1}{2}}^{-}$and $v\left(x, T+\frac{1}{2}\right) \not \equiv 0$ in $D_{T+\frac{1}{2}}^{+}$.

Solve the backward initial value problem for (4.5) with the boundary condition (4.6) and the initial condition $v\left(x, T+\frac{1}{2}\right)$ in $D_{T+\frac{1}{2}}$. Then we take $v(x, 0)$ as the initial condition $u_{0}(x)$ in (4.7) and (4.3).

Note that the map $u_{0}(x) \rightarrow v\left(x, T+\frac{1}{2}\right)$ is an open map of $\stackrel{\circ}{H_{1}}\left(D_{0}\right) \cap$ $H_{2}\left(D_{0}\right)$ to $\stackrel{\circ}{H}{ }_{1}\left(D_{T+\frac{1}{2}}\right) \cap H_{2}\left(D_{T+\frac{1}{2}}\right)$ (cf., for example, [E1]). Therefore, $v\left(x, T+\frac{1}{2}\right) \not \equiv 0$ on $D_{T+\frac{1}{2}}^{-}$and $v\left(x, T+\frac{1}{2}\right) \not \equiv 0$ on $D_{T+\frac{1}{2}}^{+}$for open dense 
set of $u_{0}(x)$, i.e. the assumption in the proof of Proposition 4.1 are satisfied for generic $u_{0}(x)$.

Remark 4.2. The electric AB effect was studied in [W2] for the electric potentials of the form $v V_{0}(v t, x)$, where $v$ is a large parameter. Note that the $\mathrm{AB}$ effect is an exact physical statement and not an asymptotic one. The introduction of a large parameter is not justified in this case.

\section{Acknowledgment.}

Author is grateful to Lev Vaidman of the Physics Department, Tel-Aviv University, and Eric Hudson of the Physics Department, UCLA, for the stimulating discussions. I am thankful to Ulf Leonhardt, St.Andrews University, for insightful remarks.

Author also grateful to the referees for valuable suggestions that significantly improved the paper.

\section{References}

[AB] Y. Aharonov and D. Bohm, Significance of electromagnetic potentials in quantum theory, Phys. Rev., 115, 485 (1959)

[BW1] M. Ballesteros and R. Weder, High-velocity estimates for the scattering operator and Aharonov-Bohm effect in three dimensions, Comm. Math. Phys., 283, 345-398 (2009)

[BW2] M. Ballesteros and R. Weder, The Aharonov-Bohm effect and Tonomura et al. experiments. Rigorous results, J. Math. Phys., 50 (2009)

[BW3] M. Ballesteros and R. Weder, Aharonov-Bohm effect and highvelocity estimates of solutions to the Schrödinger equations, Comm. Math. Phys. 303 (2011), no.1, 175-211

[E1] G. Eskin, Inverse problems for the Schrödinger equations with timedependent electromagnetic potentials and the Aharonov-Bohm effect, Journ. of Math. Phys., 49, 022105 (2008)

[E2] G. Eskin, Optical Aharonov-Bohm effect: inverse hyperbolic problem approach, Comm. Math. Phys. 284, no 2, 317-343 (2008)

[E3] G. Eskin, Inverse boundary problems and the Aharonov-Bohm effect, Inverse Problems, 19 (2003), 49-63 
[E4] G. Eskin, Inverse problems for the Schrödinger operators with electromagnetic potentials in domains with obstacles, Inverse problems, 19 (2003), 985-996

[E5] G. Eskin, Inverse boundary value problems in domains with several obstacles, Inverse Problems, 20 (2004), 1497-1516

[E6] G. Eskin, Inverse problems for Schrödinger equations with Yang-Mills potentials in domains with obstacles and the Aharonov-Bohm effect, Journal of Physics: Conference Series 12 (2005), 23-32

[EI] G. Eskin and H. Isozaki, Gauge equivalence and Inverse Scattering for Louge-Range Magnetic Potentials, Russian Journal of Math. Phys., vol. 18, No 1(2010)

[EIO] G. Eskin, H. Isozaki and S. O'Dell, Gauge equivalence and inverse scattering for Aharonov-Bohm effect, Comm. in PDE, 35, 2164-2194 (2010)

[I] V. Isakov, Carleman type estimates in an anisotropic case and applications, J. Diff. Equations, 105 (1993), 217-238

[K] Y. Kannai, Off diagonal short time asymptotics for fundamental solutions of diffusions equations, Commun. in PDE 2(1977), no. 8, 781-830

[N] F. Nicoleau, An inverse scattering problem with the Aharonov-Bohm effect, J. Math. Phys., 41, (2000), 5223-5237.

[R] S.N.M. Ruijsenaars, The Aharonov-Bohm effect and scattering theory, Annals of Phys., 146 (1983), 1-34.

[RY1] Ph. Roux and D. Yafaev, The scattering matrix for the Schrödinger operator with a long-range electro-magnetic potential, J. Math. Phys., (44) (2003), 2762-2786.

[RY2] Ph. Roux and D. Yafaev, On the mathematical theory of the Aharonov-Bohm effect, J. Phys. A: Math. Gen., 35, (2002), 7481-7492

[T et al] A. Tonomura, N. Osakabe, T. Matsuda, T. Kawasaki, J. Endo, S. Yano, and H. Yamada, Evidence for Aharonov-Bohm effect with magnetic field completely shielded from electron wave, Phys. Rev. Lett., 56, (1986), 792. 
[W1] R. Weder, The Aharonov-Bohm effect and time-dependent inverse scattering theory, Inverse Problems, 18 (2002), 1041-1056.

[W2] R. Weder, The electric Aharonov-Bohm effect, J. Math. Phys. 52 (2011), no. 5, 052109, 17 pp.

[W3] R. Weder, Inverse scattering at fixed quasi-energy for potentials periodic in time, Inverse problems 20 (2004), 893

[Y] D. Yafaev, Scattering by magnetic fields, St. Petersburg Math. J., (17) (2006), 675-695, ArXiv:math/0501544v.1. 